by Mr. Mayou. Other speakers included Mrs. Scharlieb, Dr. Macrory, Messrs. Sydney' Stephenson, Maitland Ramsay, John Wharton, A. L. Whitehead, Thomas Stevenson, Killick, and J. B. Lawford. From the point of view of State welfare, this was a most important piece of work, and we owe a debt of gratitude to those who opened and took part in the discussion. In passing a vote of thanks to the Metropolitan Asylums Board for their permission to visit St. Margaret's Hospital, the members of the Society expressed the wish to urge on the Board, now that so large a number of cases of ophthalmia neonatorum are assembled together in one institution, that facilities should be afforded at their hospital for the instruction of medical students, midwives, and district nurses in the natural history and treatment of the disease.

\title{
ANNOTATIONS
}

\section{The Pathogenesis of Detachment of the Retina}

- The pathogenesis of spontaneous or so-called idiopathic detachment of the retina has been the subject of many investigations, clinical and pathological. Indeed, if obscurity of causation can be gauged by the amount of literature, detachment of the retina occupies a high position in the scale of undetermined pathogeneses. The most recent observations are contained in an elaborate report presented to the Société française d'Ophtalmologie, last month, by Dr. Gonin (Lausanne), who was the opener of a discussion on the "Pathogenesis and Pathological Anatomy of Retinal Detachment (exclusive of that due to trauma, tumours, and parasites)." The views expressed therein recognize three pathological conditions as the chief causal agents of detachment, but their importance as determining factors is unequal. These three conditions are (1) distension of the globe, such as occurs in myopia and in hydrophthalmia, (2) traction on the retina from within, and (3) subretinal exudation. Distension of the eyeball exerts less influence on the retina itself than on the anatomical relation of the vitreous body to the retina, and on the integrity of the uveal tract; it induces nutritional changes which predispose to detachment, but does not alone give rise to it.

Traction on the retina is the determining factor in the causation of idiopathic detachment; in this process the chief role appertains to the vitreous body, but the altered conditions of this structure are themselves due to pathological changes originating in the uveal tract. Spontaneous detachment, therefore, cannot be regarded as a lesion affecting the retina alone, to the exclusion of other ocular 
tunics. This is true, also, of other non-traumatic forms of detachment. Exudation, as a causal agent, is derived from the choroid, or occurs as a serous effusion from the retina, and is the direct cause of detachment in the retinitis of albuminuria, etc. When present in idiopathic detachment such exudation plays a purely secondary part.

No real progress in the treatment of this affection, so serious and hitherto so intractable, can be hoped for unless a genuine attempt is made to determine the pathogenesis in every case.

While it is true that in many instances this is extremely difficult, it is equally true that not infrequently a thorough clinical examination affords a clue to the cause of the detachment and a guide to the measures to be advised.

\section{Ophthalmia Neonatorum}

The discussion on the prevention and treatment of ophthalmia neonatorum, which took place at the recent congress of the Ophthalmological Society of the United Kingdom, has brought into prominence once again the greatest cause of preventible blindness: Much has been done in this country to mitigate the scourge, as witness the notification of the disease, the registration of midwives, the provision of health visitors, and last, but not least, the opening in some cities, as Manchester, Liverpool, Glasgow, and London, of hospitals for the reception of affected babies and their mothers. But more is called for, and that can only be met by the adoption of prophylaxis in all cases of childbirth. The precise means adopted may differ, nor does that matter, always provided they are efficient. In the upper classes, where gonorrhoea is relatively rare, some simple means, such as that suggested by Samuel Hague in 1879 (Brit. Med. Jour., June 21, 1879, p. 959), who wiped away every trace of moisture from the baby's eyelids the moment the head was born, together with the careful washing of the baby's hands and arms, would probably suffice. In institutions where women are delivered at the cost of the community, when gonorrhoea is present in the mother, when there is a history of inflamed eyes in former babies, and in the lower classes generally, probably the best preventive is by the instillation into each eye of a single drop of 1 per cent. solution of silver nitrate. Credé, it will be recalled, employed 2 per cent. silver nitrate, and the efficacy of his method has furnished its proofs long ago. At the same time the plan is not altogether free from objections. Even when most carefully applied, it does not prevent all cases of ophthalmia. It is clearly powerless to prevent ante partum or post partum infection. Its use is nearly always followed by reaction on the part of the conjunctiva, as shown by 